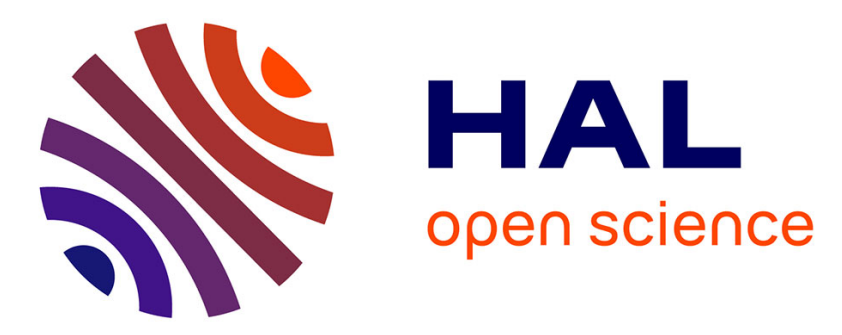

\title{
A tunable magnetic metamaterial based on the dipolar four-state Potts model
}

D. Louis, D. Lacour, M. Hehn, V. Lomakin, Thomas Hauet, F. Montaigne

\section{To cite this version:}

D. Louis, D. Lacour, M. Hehn, V. Lomakin, Thomas Hauet, et al.. A tunable magnetic metamaterial based on the dipolar four-state Potts model. Nature Materials, 2018, 17 (12), pp.1076-1080. 10.1038/s41563-018-0199-x . hal-01950274

\section{HAL Id: hal-01950274 https://hal.science/hal-01950274}

Submitted on 10 Dec 2018

HAL is a multi-disciplinary open access archive for the deposit and dissemination of scientific research documents, whether they are published or not. The documents may come from teaching and research institutions in France or abroad, or from public or private research centers.
L'archive ouverte pluridisciplinaire HAL, est destinée au dépôt et à la diffusion de documents scientifiques de niveau recherche, publiés ou non, émanant des établissements d'enseignement et de recherche français ou étrangers, des laboratoires publics ou privés. 


\section{A tunable magnetic metamaterial based on the dipolar four-state Potts model}

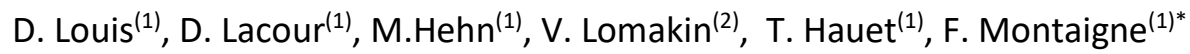

(1) Institut Jean Lamour, CNRS - Université de Lorraine, 54011 Nancy, France

(2) University of California San Diego, Department of Electrical and Computer Engineering, La Jolla, CA 92093, USA

* Corresponding author

Metamaterials, tunable artificial materials, are useful playgrounds to investigate magnetic systems. So far, artificial Ising spin systems have revealed unusual features like emergent magnetic monopoles[1,2] and charge fragmentation[3]. Here, we present a metasystem composed of a lattice of dipolarly-coupled nanomagnets. The magnetic spin of each nanomagnet is constrained to lie along a body diagonal, yielding four possible spin states. We show that the magnetic ordering of this metasystem (antiferromagnetic, ferromagnetic or spin-ice like) is determined by the spin states orientation relative to the underlying lattice. The dipolar four-state Potts model explains our experimental observations and sheds light on the role of symmetry, as well as short- and longrange dipolar magnetic interactions, in such non-Ising spin systems.

Dipolar interactions in magnetic systems have been heavily studied because of their exotic physics and strongly affect essential technologies such as permanent magnets or hard disk drives [4-6]. In addition, statistical physics covers a host of applications ranging from fundamental physics to business analysis. In both cases, Ising model holds a central role. This two-spin state model has been widely used to grasp the essence of a variety of effects belonging to the field of condensed matter physics, such as phase transitions, spin glasses, magnetism, spin chains and geometric frustration. In 2006, Zhang and collaborators conducted the first experiments on artificial Ising spin systems at a 
mesoscopic scale. Their findings were based on the exact determination of the spin configurations. This was made possible by the use of nano-magnets having a uniaxial anisotropy mimicking the two spin state of the Ising model. This approach proved to be particularly fruitful, especially to study original spin ices phenomena [1-3,7-9].

So far, artificial spin systems have been restricted to the Ising variety, i.e., the two spin states being associated with the two possible magnetization states of a nanomagnet. However, beyond the Ising models, other spin lattice models, such as Potts, XY, Heisenberg or more generally $n$-vector models, have been theoretically studied and recognized to be of interest beyond the purely academic point of view. In particular, the Potts model [10] has found applications in many complex systems [11-13]. Here, we introduce a variant of the Potts model: the dipolar four-state Potts model. This model system is experimentally produced as an array of artificial Potts spins, i.e. an array of dipolar coupled nanomagnets having four equivalent states of magnetization.

Artificial spins must meet several specifications. An important specification is the mapping between the micromagnetic configurations and the desired spin model. The magnetic anisotropy of the nanostructure is the key ingredient to ensure a mostly uniform magnetization state within the nanostructure. This is important to prevent non-homogenous equilibrium magnetization configurations, as they can lead to the appearance of internal degrees of freedom $[14,15]$; this increases the complexity of the system under study, and thus deviates away from the ideal spin model. Up to now, artificial Ising spins have been implemented through uniaxial anisotropy induced either by the shape of the elements (in-plane magnetization [16-18]) or by the material anisotropy (out of plane magnetization [19-20]).

Our four-states Potts artificial spins are made of a magnetic thin film having an in-plane magnetization and a well-defined cubic anisotropy [21]. A $2 \mathrm{~nm}$ thin epitaxial iron film is shaped in $300 \mathrm{~nm}$ wide squares having their diagonals aligned with the [100] and [010] anisotropy directions, 
the in-plane easy axes of the Fe layer (fig. 1). At this thickness, the demagnetizing energy (scaling as the square of the thickness) is reduced enough to avoid the existence of a non-uniform ground state [22-24]. This geometry ensures a quasi-uniform ground state without any internal degrees of freedom and two equivalent directions with four possible stable states [25] along the square diagonals. Moreover, in this thickness range, these artificial spins can be thermally excited (see SI1). This allows driving the system from a paramagnetic-like state toward the ground state by use of an external thermal bath as done in the case of artificial Ising spins systems $[7,8,26]$.

We focus here on artificial Potts spins on square lattices. While the element diagonals remain aligned with the Fe crystal to ensure the aforementioned magnetic properties, the lattice orientation can be varied. This provides a particularly interesting tuning knob as shown in the following parts. Figures 1. $d, e, f$ represent the different geometries studied, defined by $\alpha$, the angle between spins, and lattice direction; $\alpha$ taking values equal to $0^{\circ}, 22.5^{\circ}$ and $45^{\circ}$.
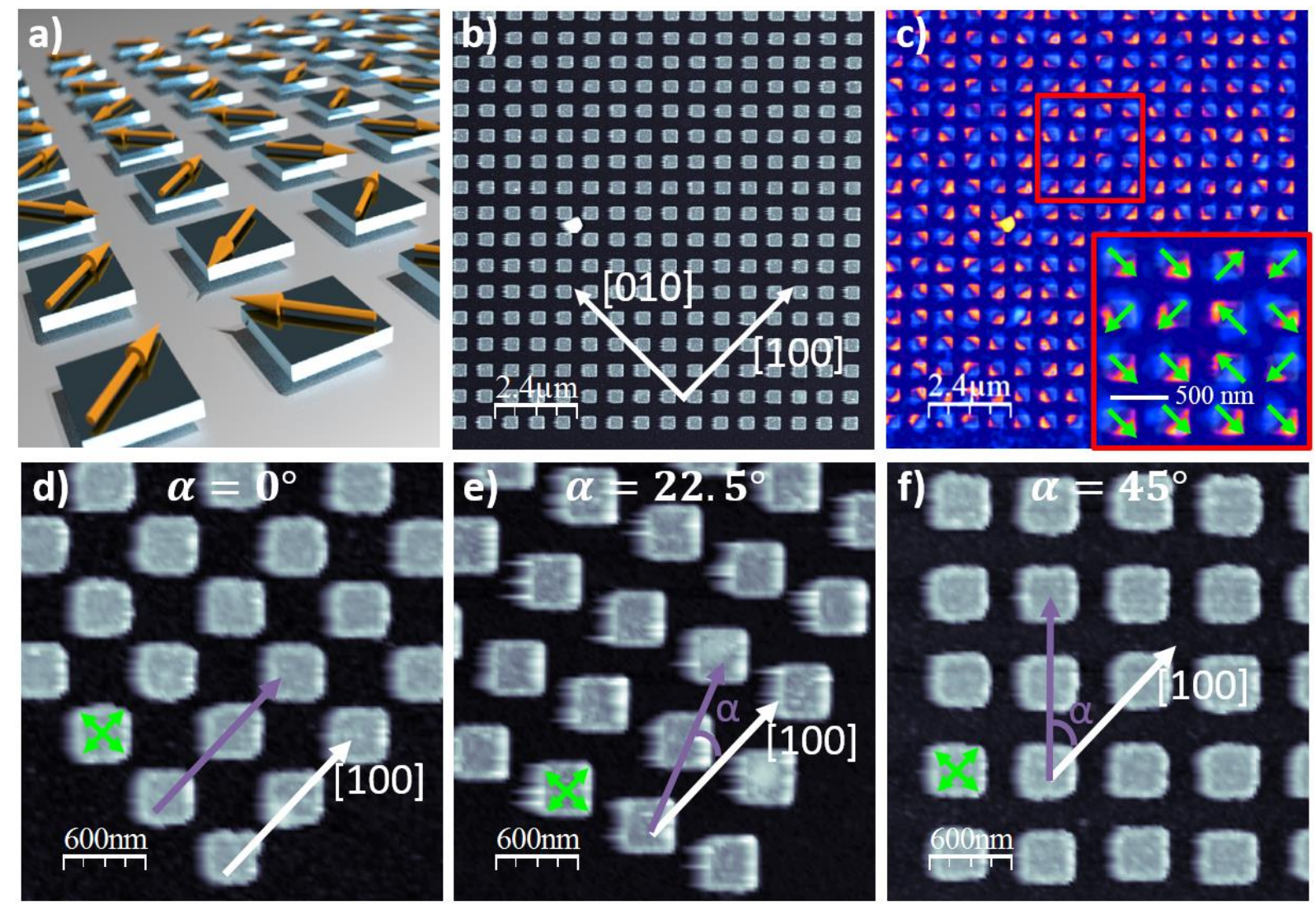

Fig. 1 | Presentation of the system and geometry definition. a, schematic diagram of the artificial spin system. b, Atomic Force Microscopy (AFM) image of a portion of the lattice. c, Corresponding Magnetic Force Microscopy (MFM) image. The zoom indicates the correspondence between the MFM contrast and the spin value. d-f, AFM images describing the three 
The state of each four-state Potts artificial spin can be determined directly by Magnetic Force Microscopy (MFM) as exemplified in figure 1.c. The magnetic configurations are stable at room temperature and not affected by the MFM measurement (SI1). However, $350{ }^{\circ} \mathrm{C}$ annealing leads to switch of spins towards one of the four accessible directions (SI1).

Figures 2. $(a, b, c)$ represent the magnetic configurations obtained after thermal treatment for the three lattice directions $\left(\alpha=0^{\circ}, 22.5^{\circ}\right.$ and $\left.45^{\circ}\right)$ having an identical period of $500 \mathrm{~nm}$ and a $30 \times 30$ size. A usual approach to get insight into magnetic order consists in considering the magnetic structure factor as measured in neutron scattering experiments, which can be computed from the exact magnetization configurations [9]. The magnetic structure factors associated with the three presented magnetization configurations bring to light the existence of different magnetic order (SI2). Going back to the real space, it is indeed possible to ascribe a large part (from 87 to $94 \%$ ) of the magnetic configurations to four different local orders: (i) Ferromagnetic order in which all the spins have the same values (red color in figure 2). (ii) Collinear antiferromagnetic order with alternation of lines of opposite spins (cyan color in figure 2). (iii) Spin-ice order that is identical to the type I vertex introduced by Wang et al. [16] for the square Ising spin ice (green color in figure 2), which corresponds to a vertex obeying the "ice rules" with two spins pointing in and two spins pointing out. Spin-ice domains can also be seen as crystals of spin loops with alternating chiralities. (iv) Zigzag order corresponds to lines of spins being perpendicular to one another in one direction and parallel in the other direction (magenta color in figure 2). It can be seen as a mixture of ferromagnetic and spin-ice orders. 


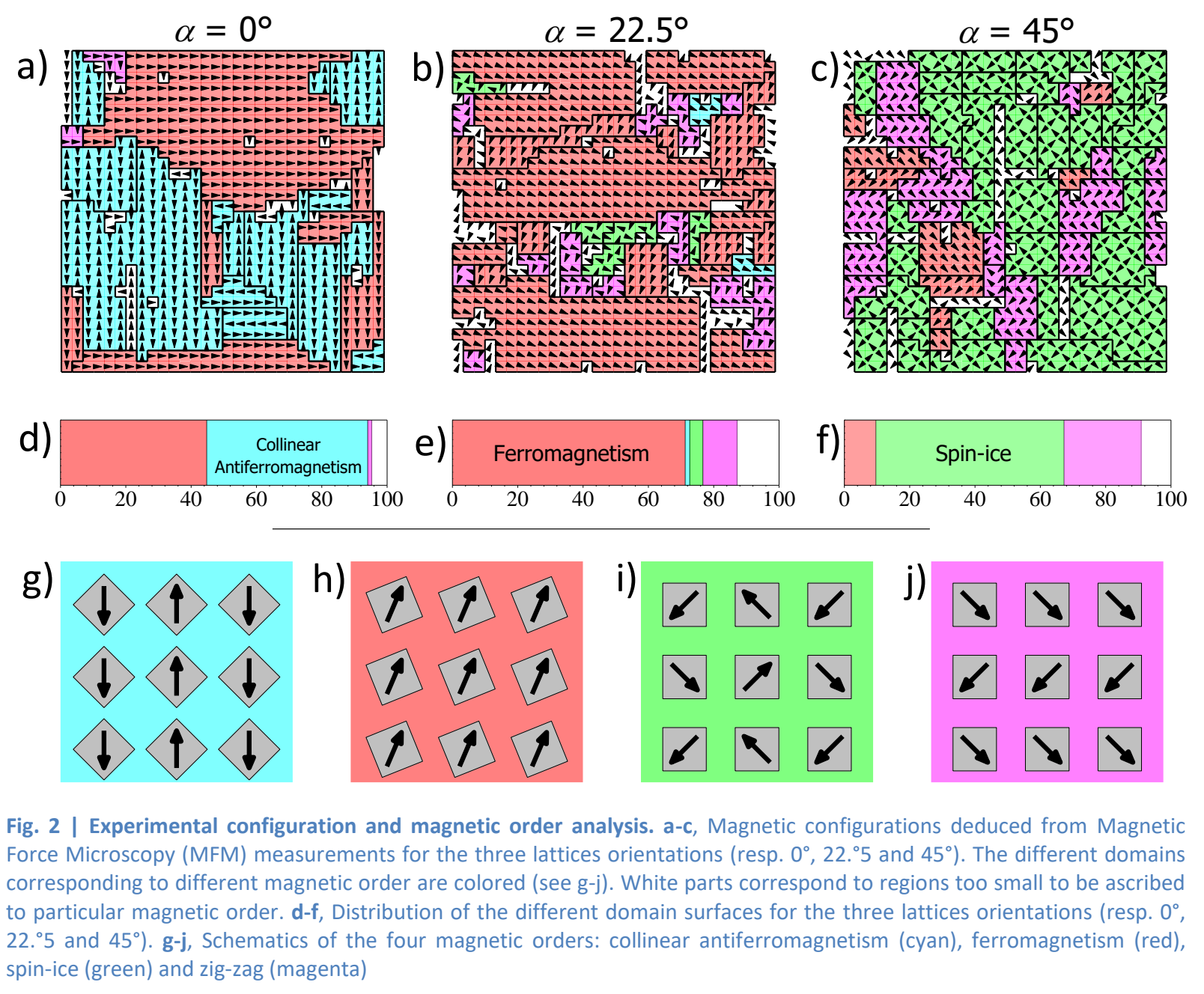
spin-ice (green) and zig-zag (magenta)

As evident from figure 2, whereas both the lattice and the spins are unchanged, the magnetization configurations depend drastically on their relative orientation $\alpha$. For spin directions along the lattice directions $\left(\alpha=0^{\circ}\right)$, a large fraction of antiferromagnetic domain is observed (49\% of the surface). The collinear antiferromagnetic order is easily understood by considering the dipolar interaction between the nearest neighbors and results from the anisotropic nature of the dipolar coupling. For spin oriented along the lattice diagonals $\left(\alpha=45^{\circ}\right)$, no antiferromagnetic domain is observed but spin ice domains are present ( $58 \%$ of the surface). Similar to the conventional square ice with Ising spins [16] this specific configuration is deduced from considerations of local interactions. For an intermediate angle, $\alpha=22.5^{\circ}$, large ferromagnetic domains are observed. This local order cannot be explained considering nearest-neighbor interactions (which would promote the two previous states). This is 
rather counter-intuitive as dipolar interactions in 2D and 3D systems generally destabilize ferromagnetism. A quantitative analysis of the dipolar four-state Potts model is thus necessary.

The dipolar four-state Potts model differs drastically from the usual Potts models (clock or standard [10]). Whereas the spin nature is the same within the dipolar four-state Potts model, the interactions between spins are anisotropic and cannot be described only by a function of spins values like $\cos \left(\theta_{i}-\theta_{j}\right)$ or $\delta_{\theta_{i}, \theta_{j}}$. The total Hamiltonian of the system can be expressed as:

$$
H=\frac{\mu_{0} \cdot m^{2}}{N} \sum_{i<j}^{N} \frac{3\left(\boldsymbol{S}_{\boldsymbol{i}} \cdot \boldsymbol{e}_{\boldsymbol{i j}}\right)\left(\boldsymbol{S}_{\boldsymbol{j}} \cdot \boldsymbol{e}_{\boldsymbol{i} \boldsymbol{j}}\right)-\boldsymbol{S}_{\boldsymbol{i}} \cdot \boldsymbol{S}_{\boldsymbol{j}}}{r_{i j}{ }^{3}}
$$

where $\boldsymbol{e}_{\boldsymbol{i} \boldsymbol{j}}$ is a unit vector between sites $i$ and $j, m$ is the magnetic moment of the spins, and $N$ is the number of spins. To emphasize the specificity of the dipolar Potts model and its angular dependence, it is interesting to consider the interaction between two spins. For two Ising spins, this interaction results in two energy levels (figure 3.a) and, depending on the angle between the spin direction and their relative position $\left(\alpha=\cos ^{-1} \boldsymbol{S}_{\boldsymbol{i}} \cdot \boldsymbol{e}_{\boldsymbol{i}}\right)$, the coupling is either ferromagnetic $\left(\alpha<54.7^{\circ}\right)$ or antiferomagnetic $\left(\alpha>54.7^{\circ}\right)$. For a given value of $\alpha$, it is possible to describe the interaction by a single scalar $J_{i j}$ such that the energy writes $-J_{i j} . \sigma_{i} . \sigma_{j}$ with $\sigma_{i, j}= \pm 1$. For the four-state dipolar Potts model, the 16 different possible configurations result in 6 different energy levels (figure 3.b) and the interaction cannot be described anymore by a single scalar. The lowest energy level is either parallel $\left(\alpha<29.3^{\circ}\right)$ or perpendicular $\left(\alpha>29.3^{\circ}\right)$ and can also be degenerate $\left(\alpha=29.3^{\circ}\right)$. 

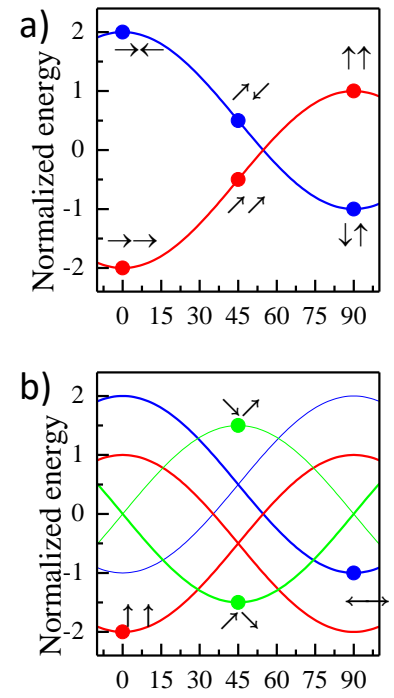

Spin orientation $\alpha$ (deg.)

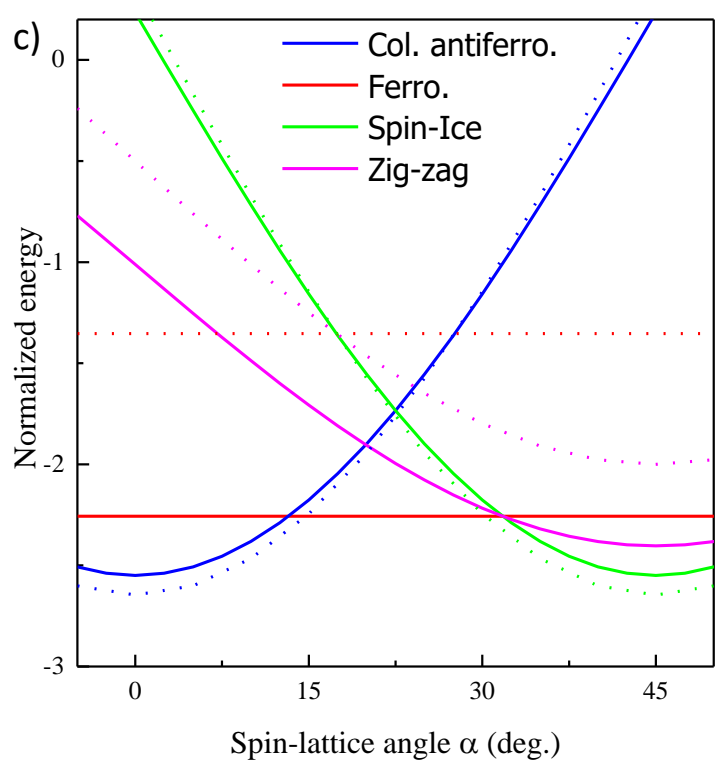

Fig. 3 | Energy angular dependence. a,b, Energy levels for two Ising spins as a function of the angle made between the spins and the direction between them, 2 possible configurations result in 2 energy levels (a) and two Potts spins, 16 possible configurations result in 6 energy levels (b). c, Energy per spin for ferromagnetic state (red), collinear antiferromagnetic state (blue), spin-ice state (green) and zigzag state (magenta). Dotted lines correspond to first neighbor interactions and continuous lines to all interactions. All energies are normalized to $\mu_{0} . m^{2} / a^{3}, \mu_{0}$ being the vacuum permeability, $\boldsymbol{m}$ the magnetic moment of an element and $\boldsymbol{a}$ the closest distance between spins.

For the case of the square lattice, the discussion is focused on the four typical configurations experimentally observed. Figure 3.c represents with dotted lines the energy of these configurations considering only the nearest-neighbor interactions (short range, 8 closest neighbors) for an infinite lattice (periodic boundary conditions). The collinear antiferromagnetic and the spin ice configurations appears to be the lowest energy configuration for $\alpha$ inferior and superior to $22.5^{\circ}$, respectively. The ferromagnetic configuration (dotted red line of figure 3 ) observed predominantly at $22.5^{\circ}$ is not the ground state of the short-range model. The same conclusions hold if only the 4 closest neighbors are considered, the difference between energy levels being larger. However, taking into account all longrange dipolar interactions (solid lines in figure 3.c,) leads to a reduction of the energy of the ferromagnetic state (solid red line of figure 3), which becomes the ground state for an angular range around $22.5^{\circ}$. On the other hand, taking into account all long-range dipolar interactions does not significantly affect the configurations with no net magnetization. Therefore, we find that the nature of the ground state depends strongly on the angle $\alpha$ between spins and lattice. For spin directions close to the lattice directions $\left(|\alpha|<13.2^{\circ}\right)$, the lowest energy state is the collinear antiferromagnetic 
state. For spin directions close to $45^{\circ}$ from the lattices directions $\left(|\alpha-45|<13.2^{\circ}\right)$, the lowest energy state is the ordered spin-ice state. For intermediate spin directions $\left(13.2<\alpha<31.8^{\circ}\right)$, it is the ferromagnetic order that has the lowest energy state. Monte-Carlo simulations were used to confirm that these states are indeed the lowest energy ones for an infinite lattice. For finite lattice size, the same conclusions hold but there exist multi-domain configurations which further decreases the energy of ferromagnetic state. Note that the energy of the zigzag state is the average between the one of the ferromagnetic and spin-ice states, and the zig-zag state is the ordered state with energy just beyond the ground state for $|\alpha-45|<22.6^{\circ}$. This theoretical analysis is based on the point dipole approximation. However, this approximation is questionable in our experimental case of nanomagnets with lateral extension not negligible compared to the lattice period $(300 \mathrm{~nm}$ vs $500 \mathrm{~nm}$ ). Therefore, we show in SI 3 with micromagnetic simulations that actually taking into account the magnetization distribution within the elements and their spatial extension, does not affect the previous conclusions.

From these main features of the dipolar four-state Potts model, it is possible to quantitatively analyze the experimental data. For the configurations represented in figure 2 , the proportion of antiferromagnetic domains in the sampled area is $44 \%$ for $\alpha=0^{\circ}$, the proportion of ferromagnetic domains is $73 \%$ for $\alpha=22.5^{\circ}$, and the proportion of spin-ice domains is $64 \%$ for $\alpha=45^{\circ}$. These proportions are significant and well above the statistical noise level (which is below $8 \%$ for the sample size investigated here) and correspond to large ground-state domains. These experimental results are in agreement with our theoretical predictions.

The general behavior of the presented breed of artificial spin system is, therefore, well-explained within our theoretical framework. Beyond this analysis, as in many systems, the domain (or crystallite) structure reveals underlying mechanisms. It appears that the thermal procedure we used did not drive all the spins into a superparamagnetic state, as reminiscence of the initial state (saturated) are clearly observed for $\alpha=0$ and $22.5^{\circ}$. This reminiscence is due to the low energy of 
this ferromagnetic configuration and not to properties of individual spins. For instance, in the case of $\alpha=22.5^{\circ}$, the proportion of ferromagnetic domains drops from $73 \%$ to $20 \%$ when the period of the lattice is increased from 500 to $700 \mathrm{~nm}$, which corresponds to the reduction of the dipolar interactions by a factor of 2.7 (see SI4). The high temperature dynamics is also revealed by the orientation of the configurations. For $\alpha=0^{\circ}$ (figure 2.a), the antiferromagnetic domains are mostly aligned perpendicularly to the initial saturation direction. For $\alpha=45^{\circ}$, the size of the spin-ice domains is limited by the number of spins opposite to the initial saturation direction, which is quite small at $12 \%$. This indicates that the reversal of spins proceeds by a $90^{\circ}$ rather than $180^{\circ}$ rotation. The presented thermal procedure is therefore not fully efficient. The system does not explore the entire energy landscape and is trapped in a local energy minimum. Nevertheless, the system can be driven in domains of the expected low energy configurations. It is interesting to compare the energy scales of the four-state dipolar Potts model at $45^{\circ}$ from a square lattice and the most studied square Ising ice. The ground state is identical for both systems (type I vertices). It can be reached almost perfectly in the square Ising ice via a superparamagnetic regime [26]. But the energy difference between the two lowest energy states (type I and type II vertexes) is about 10 times the one between the two lowest energy states of the Potts system (considering a dipolar approximation). Achieving a perfect thermalization of the dipolar Potts system remains a challenge.

In conclusion, the dipolar four-state Potts square lattice is a versatile system; since a relative rotation between spin and lattice directions gives rise to very different properties and particularly different ground states. Interestingly, at $\alpha=22.5^{\circ}$, a ferromagnetic phase originates only from dipolar interactions. This is a striking result since ferromagnetism is generally associated with short-range exchange interactions in a 2- or 3-dimensional systems, whereas long range dipolar interactions tend to decrease the net magnetic moment. The four state dipolar Potts model, therefore, adds up to the short list of 2D systems exhibiting dipolar ferromagnetism [27,28]. The spin-ice phase is an alternative realization of artificial spin ice. Its ground state is identical to the square ice proposed by 
Wang et al. [16], following a two in / two out ice rule (type 1 vertex), but the number of different vertex type is much higher, being 15 instead of 4 . More generally, the four-state dipolar Potts model offers an opportunity to precisely tune (via a geometrical parameter) the energy levels of the system. At $\alpha=31.8^{\circ}$ the ground state is degenerated with several ordered phases having the same energy. The work presented here can be viewed as the first experimental realization of the dipolar four-state Potts model with artificial spins made of nanomagnets, which shows that main features of the theoretical model can be probed experimentally. We believe that this concept can be applied more generally to a host of other lattice types.

Beyond these fundamental aspects, we have shown that the system behaves like a magnetic metamaterial having an easily tunable magnetic order. Our experimental model system is based on thin epitaxial films but the concept can be extended with other nano-objects exhibiting a cubic anisotropy. Nano-objects with shape-driven anisotropy defined by lithography are interesting but bottom-up approaches relying on chemical synthesis are very promising $[29,30]$ 


\section{References}

[1] Ladak, S., Read, D. E., Perkins, G. K., Cohen, L. F. \& Branford, W. R. Direct observation of magnetic monopole defects in an artificial spin-ice system. Nature Phys. 6, 359-363 (2010).

[2] Mengotti, E. et al. Real-space observation of emergent magnetic monopoles and associated Dirac strings in artificial kagome spin ice. Nature Phys. 7, 68-74 (2011).

[3] Canals, B. et al. Fragmentation of magnetism in artificial kagome dipolar spin ice. Nat. Commun. 7, 11446 (2016).

[4] Schrefl T., Schmidts H.F., Fidler J. Kronmüller H., The role of exchange and dipolar coupling at grain boundaries in hard magnetic materials, J. Magn. Magn. Mater. 124, 251 (1993).

[5] Miller J.S., Drillon M., Magnetism: Molecules to Materials IV: Nanosized Magnetic Materials, book chapter 7, Wiley (2003).

[6] Majetich, S. A., Sachan, M., Magnetostatic interactions in magnetic nanoparticle assemblies: energy, time and length scales, J. Phys. D 39, 407 (2006).

[7] Anghinolfi, L. et al. Thermodynamic phase transitions in a frustrated magnetic metamaterial. Nature Communications 6, 9278 (2015).

[8] Gilbert, I. et al. Emergent reduced dimensionality by vertex frustration in artificial spin ice. Nat. Phys. 12, $162(2016)$.

[9] Perrin, Y., Canals, B. \& Rougemaille, N. Extensive degeneracy, Coulomb phase and magnetic monopoles in artificial square ice. Nature 540, 410-413 (2016).

[10] Wu, F.Y., The Potts model. Rev. Mod. Phys. 54, 235 (1982)

[13] Sanyal, S. \& Glazier, J. A. Viscous instabilities in flowing foams: a Cellular Potts Model approach. J Stat Mech p10008 (2006).

[12] Sun, L., Chang, Y. F. \& Cai, X. A discrete simulation of tumor growth concerning nutrient influence. Int. J. Mod. Phys. B 18, 2651-2657 (2004).

[11] Schelling, T. C. Dynamic models of segregation. The Journal of Mathematical Sociology 1, 143-186 (1971).

[14] Rougemaille, N. et al. Chiral nature of magnetic monopoles in artificial spin ice. New Journal Of Physics 15 , 035026 (2013) 
[15] Gliga, S., Kakay, A., Heyderman, L.J., Hertel, R., Heinonen, O.G. Broken vertex symmetry and finite zeropoint entropy in the artificial square ice ground state. Phys. Rev. B 92(6), 060413 (2016).

[16] Wang, R. F. et al. Artificial 'spin ice' in a geometrically frustrated lattice of nanoscale ferromagnetic islands. Nature 439, 303-306 (2006).

[17] Qi, Y., Brintlinger, T. \& Cumings, J. Direct observation of the ice rule in an artificial kagome spin ice. Phys. Rev. B 77, 094418 (2008).

[18] Arnalds, U. B. et al. A new look on the two-dimensional Ising model: thermal artificial spins. New J. Phys. 18,023008 (2016).

[19] Zhang, S. et al. Perpendicular magnetization and generic realization of the Ising model in artificial spin ice. Phys. Rev. Lett. 109, 087201 (2012).

[20] Chioar, I. A. et al. Nonuniversality of artificial frustrated spin systems. Phys. Rev. B 90, 064411 (2014).

[21] Louis, D. et al. Interfaces anisotropy in single crystal V/Fe/V trilayer. J. Magn. Magn. Mater. 372, 233-235 (2014).

[22] Vaz, C.A.F. et al. Direct observation of remanent magnetic states in epitaxial fcc Co small disks. Phys. Rev. B $67,140405(R)(2003)$

[23] Mitsuzuka, K., Lacour, D., Hehn, M., Andrieu, S. \& Montaigne, F. Magnetic vortices in single crystalline Fe-V disks with four folds magnetic anisotropy. Appl. Phys. Lett. 100, 192406 (2012).

[24] Li, J. et al. Stabilizing a magnetic vortex/antivortex array in single crystalline $\mathrm{Fe} / \mathrm{Ag}(001)$ microstructures. Appl. Phys. Lett. 104, 262409 (2014).

[25] Louis, D. et al. Four states magnetic dots: A design selection by micromagnetic modeling. in Spintronics IX (eds. Drouhin, H. J., Wegrowe, J. E. \& Razeghi, M.) 9931, UNSP 99311M (Spie-Int Soc Optical Engineering, 2016). [26] Farhan, A. et al. Direct observation of thermal relaxation in artificial spin ice. Phys. Rev. Lett. 111, 057204 (2013).

[27] Puntes, V. F., Gorostiza, P., Aruguete, D. M., Bastus, N. G. \& Alivisatos, A. P. Collective behaviour in twodimensional cobalt nanoparticle assemblies observed by magnetic force microscopy. Nat Mater 3, 263-268 (2004).

[28] Yamamoto, K. et al. Direct visualization of dipolar ferromagnetic domain structures in Co nanoparticle monolayers by electron holography. Appl. Phys. Lett. 93, 082502 (2008). 
[29] Sun, S.H., Murray, C.B., Weller, D., Folks, L., Moser, A., Monodisperse FePt nanoparticles and ferromagnetic FePt nanocrystal superlattices. Science 287, 1989-1992 (2000)

[30] Yang, W., Yu, Y., Wang, L., Yang, C., Li, H., Controlled Synthesis and Assembly into Anisotropy Arrays of Magnetic Cobalt-Substituted Magnetite Nanocubes. Nanoscale 7, 2877-2882(2015)

\section{Acknowledgements}

The authors want to thank N. Rougemaille and B. Canals for fruitful discussions. This work was supported by the Agence Nationale de la Recherche through project number ANR12-BS04-009 'Frustrated' and partially supported by the French PIA project "Lorraine Université d'Excellence" ANR15-IDEX-04-LUE.

\section{Author contributions}

Sample preparation: D.Lo., T.H, M.H., F.M. ; MFM measurements: D.Lo, D.La, M.H. ; Data analysis: D.Lo, D.La, F.M. ; Theory and simulations: D.Lo, F.M., V.L. ; All authors discussed the results and implications at all stages and prepared the manuscript.

\section{Competing interests}

The authors declare no competing financial interest

\section{Data availability statement}

The data that support the findings of this study are available from the corresponding author upon reasonable request. 


\section{Methods}

\section{Sample fabrication}

The $\mathrm{MgO} / / \mathrm{V}(50 \mathrm{~nm}) / \mathrm{Fe}(2 \mathrm{~nm}) / \mathrm{V}(4 \mathrm{~nm}) / \mathrm{Au}(4 \mathrm{~nm})$ multilayer were grown by Molecular Beam Epitaxy as described in [21]. The magnetic elements were patterned by ebeam lithography (P(MAAMMA)/PMMA bilayer resist) and lift-off of an aluminum film (60 $\mathrm{nm})$. The pattern was transferred by Ion Beam Etching down to the vanadium buffer layer. To reduce the interaction between the MFM tip and the magnetic elements, a $10 \mathrm{~nm}$ aluminum film was deposited over the entire surface by sputtering.

\section{Thermal treatment and Magnetic Force Microscopy}

The sample was heated at $350^{\circ} \mathrm{C}$ for 5 hours in vacuum. The MFM measurements were done with a lift height of about $25 \mathrm{~nm}$ on a Dimension 3100 nano-scope equipped with Quadrex module for phase detection. Asylum low-moment tips were used to minimize the sample-tip magnetic interactions. 